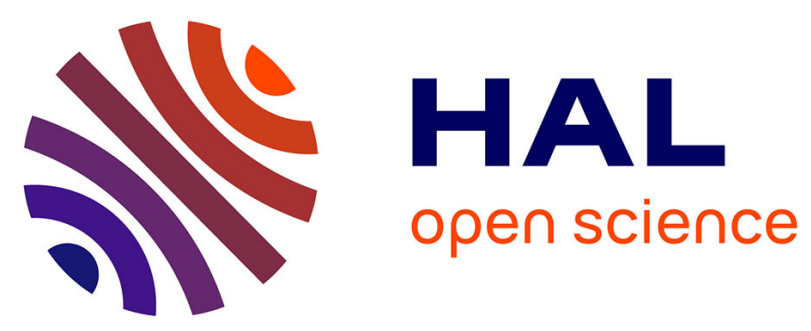

\title{
To what extent can cortical bone millimeter-scale elasticity be predicted by a two-phase composite model with variable porosity?
}

Mathilde Granke, Quentin Grimal, William J. Parnell, Kay Raum, Alf Gerisch, Françoise Peyrin, Amena Saïed, Pascal Laugier

\section{To cite this version:}

Mathilde Granke, Quentin Grimal, William J. Parnell, Kay Raum, Alf Gerisch, et al.. To what extent can cortical bone millimeter-scale elasticity be predicted by a two-phase composite model with variable porosity?. Acta Biomaterialia, 2015, 12 (15), pp.207-215. 10.1016/j.actbio.2014.10.011 . hal-01126647

\section{HAL Id: hal-01126647 \\ https://hal.science/hal-01126647}

Submitted on 13 Apr 2016

HAL is a multi-disciplinary open access archive for the deposit and dissemination of scientific research documents, whether they are published or not. The documents may come from teaching and research institutions in France or abroad, or from public or private research centers.
L'archive ouverte pluridisciplinaire $\mathbf{H A L}$, est destinée au dépôt et à la diffusion de documents scientifiques de niveau recherche, publiés ou non, émanant des établissements d'enseignement et de recherche français ou étrangers, des laboratoires publics ou privés. 


\section{To what extent can cortical bone millimeter scale elasticity be predicted by a two phase composite model with variable porosity?}

Mathilde Grankea, , Quentin Grimala ${ }^{\mathrm{a}, \mathrm{b}}$, William J Parnell ${ }^{\mathrm{c}}$, Kay Raum ${ }^{\mathrm{d}}$, Alf Gerisch ${ }^{\mathrm{e}}$, Françoise Peyrin ${ }^{\mathrm{f}, \mathrm{g}}$, Amena Saïed ${ }^{\mathrm{a}, \mathrm{b}}$, and Pascal Laugier ${ }^{\mathrm{a}, \mathrm{b}}$

aUPMC Univ Paris 6, UMR 7623, Laboratoire d’Imagerie Paramétrique, 75005 Paris, France

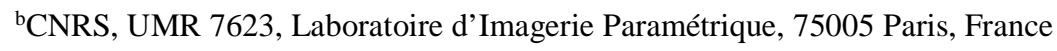

${ }^{c}$ School of Mathematics, Alan Turing Building, University of Manchester, M139PL Manchester, UK

dJulius Wolff Institute \& Berlin-Brandenburg School for Regenerative Therapies, Charité-Universitätsmedizin

Berlin, 13353 Berlin, Germany

eFachbereich Mathematik, Technische Universität Darmstadt, 64289 Darmstadt, Germany

fESRF, 6 rue Jules Horowitz, 38043 Grenoble, France

gCREATIS INSERM U1044, CNRS 5220; INSA Lyon; Université de Lyon, 69621 Villeurbanne, France

\section{Corresponding author:}

\section{Mathilde Granke}

Present address:

Vanderbilt Orthopaedic Institute

Medical Center East, South Tower, Suite 4200

Nashville, TN 37232-8774 USA

tel: +1 615554 3035; email: mathilde.granke@gmail.com 


\section{Abstract}

2 An evidence gap exists in fully understanding and reliably modeling the variations in elastic

3 anisotropy that are observed at the millimeter scale in human cortical bone. The porosity (pore

4 volume fraction) is known to account for a large part, but not all, of the elasticity variations.

5 This effect may be modeled by a two-phase micromechanical model consisting of a

6 homogeneous matrix pervaded by cylindrical pores. Although this model has been widely

7 used, it lacks experimental validation. The aim of the present work is to revisit experimental

8 data (elastic coefficients, porosity) previously obtained from 21 cortical bone specimens from

9 the femoral mid-diaphysis of 10 donors and test the validity of the model by proposing a detailed discussion of its hypotheses. This includes investigating to what extent the

11 experimental uncertainties, pores network modeling, and matrix elastic properties influence the model's predictions. The results support the validity of the two-phase model of cortical bone which assumes that the essential source of variations of elastic properties at the

14 millimeter-scale is the volume fraction of vascular porosity. We propose that the bulk of the

15 remaining discrepancies between predicted stiffness coefficients and experimental data

16 (RMSE between 6\% and 9\%) is for a part due to experimental errors and for another part due

17 to small variations of the extravascular matrix properties. More largely, although most of the

18 models that have been proposed for cortical bone were based on several steps of

19 homogenization and a large number of variable parameters, we show that a model with a

20 single parameter, namely the volume fraction of vascular porosity, is a suitable representation

21 for cortical bone. The results could provide a guide to build specimen-specific cortical bone

22 models. This will be of interest to analyze the structure-function relationship in bone and to 23 design bone mimicking materials.

24 Keywords: mechanical model; anisotropic elasticity; cortical bone; effective properties;

25 porosity 


\section{Introduction}

2 Bone is a multiscale biocomposite whose structure and mechanical properties at one level

3 determine the properties of the subsequent one. Despite numerous studies dedicated to the

4 assessment of cortical bone mechanical properties, some questions remain open regarding the

5 determinants of cortical bone elastic properties which are known to vary, among others, with

6 age, anatomic location, disease, or drug treatment. A clear understanding as well as a good

7 representation of the elastic properties and their variations is needed for the modeling of the

8 macroscopic (organ scale) behavior of bones, the investigation of structural-functional

9 relationships (remodeling) or the design of new in vivo techniques to monitor bone properties.

10 At the mesoscale (2-10 millimeters [1]), cortical bone can be described as a two-phase

11 composite material consisting of a dense mineralized matrix and a soft phase, i.e. Haversian

12 canals, Volkmann's canals, and resorption cavities (referred to as vascular porosity)

13 containing fluid and soft tissues. The porosity has been established to be an important

14 determinant of the mesoscopic bone properties [2-4]. On the other hand, considering only

15 published experimental studies in human cortical bone, the impact of the bone matrix elastic

16 properties (i.e. at the microscopic level) on bone mesoscale elasticity is a matter of debate in

17 the literature $[3,5]$. In a previous experimental study [6], we addressed the question of the

18 respective contributions of the variations of porosity and bone matrix elasticity (reflected by

19 acoustical impedance) to changes of mesoscopic elastic properties. We found that the elastic

20 properties of the matrix only undergo small variations among different specimens

21 (coefficients of variation of matrix impedance values were less than 6\%) and that variations in

22 porosity account for most of the variations of mesoscopic elasticity, at least when the

23 analyzed porosity range is large (3-27\%). These results suggest that, in a first approach, the

24 variations of mesoscale cortical stiffness could be modeled by a simple micromechanical

25 model where the matrix would be the same for all bone specimens (i.e., fixed matrix stiffness 
1 coefficients) and the porosity would be the only specimen-dependent parameter. A reasonable

2 model, already proposed by several authors [7-10], consists of a two-phase micromechanical

3 model: a homogeneous matrix with transversely isotropic stiffness pervaded by cylindrical

4 pores aligned with the direction of highest matrix stiffness. This two-phase model, when

5 implemented with fixed matrix properties (further referred to as the reference model),

6 correctly predicts the trend of the variation of each elastic coefficient as a function of the

7 porosity [6]. However, there remain unexplained discrepancies between the predicted and

8 measured stiffness coefficients for most of the specimens. These discrepancies may originate

9 from different sources. On the one hand, the two-phase model is only a rough idealization of

10 bone: the modeled porosity is uniformly distributed and the pores are circular, regular and

11 infinitely long; on the other hand, the experimental data (evaluation of stiffness and vascular

12 porosity) is subject to several measurement errors.

13 The objective of the present paper is to test the validity of the reference model (matrix

14 pervaded by cylindrical pores) by proposing a detailed discussion of its hypotheses and to

15 determine to what extent cortical bone millimeter scale anisotropic elasticity can be predicted

16 based on the sole knowledge of porosity. One added value of this study is the systematic

17 quantification of all the potential sources of discrepancies that could be modeled and the

18 discussion of their relative contributions. One further originality of our work is that we

19 compare the predictions of a popular category of micromechanical models accounting only

20 for pore volume fraction and the predictions of a finite element (FE) model which accounts

21 for the distribution of the pore volume fraction within the cortical specimen.

22 This paper is organized as follows. Section 2 briefly presents the experimental findings of

23 Granke et al. [6] and the reference two-phase model. Section 3 quantifies the discrepancies

24 between data and model predictions. We then clarify how far the experimental data can be

25 trusted (section 4) before revisiting the hypotheses of the model to search for factors, besides 
1 changes in pore volume fraction, that would explain the discrepancies between data and

2 model predictions (section 5).

3

\section{Experimental data and reference model}

5 The specimens preparation and measurement methods, which were described in detail in

6 Granke et al. [6] are summarized below. The data used in the present study was obtained on

721 parallelepiped specimens (nominal size $5 \times 5 \times 7 \mathrm{~mm}^{3}$ ) from 10 female donors (mean age

881 years, range 66-98 years). The faces of the specimens were oriented according to the radial

9 (1), circumferential (2), and longitudinal (3) axes defined by the anatomic shape of the

10 femoral diaphysis. The diagonal terms of the apparent (i.e., mesoscopic) stiffness tensor -

11 longitudinal $\left(C_{11}, C_{22}, C_{33}\right)$ and shear $\left(C_{44}, C_{55}, C_{66}\right)$ elastic coefficients - were determined

12 from the apparent mass density and wave velocity measurements using a well-established

13 pulse transmission method [11]. The vascular porosity $P$ was obtained from 50-MHz-

14 scanning acoustic microscopy (SAM $[12,13])$ with a resolution of 30 microns. The 3D pore

15 network was imaged with a resolution of $10 \mu \mathrm{m}$ for a subset of 10 specimens using

16 synchrotron radiation micro-computed tomography (SR- $\mu \mathrm{CT}$, ESRF, Grenoble, France).

17 The reference two-phase model predicts mesoscopic elastic properties exclusively accounting

18 for variations of the pore volume fraction. Different homogenization schemes have been used

19 by different authors to calculate the predictions of such a model: asymptotic homogenization

20 [7], Mori-Tanaka method [9], generalized self-consistent method [8]. Our implementation of

21 the reference model uses asymptotic homogenization $(\mathrm{AH})[14,15]$. The model hypothesizes

22 that cortical bone can be regarded as a homogeneous transversely isotropic (TI) matrix

23 pervaded by infinite cylindrical pores, periodically distributed within the matrix material

24 (specifically on a hexagonal lattice). An orthonormal Cartesian frame $\left(\mathbf{x}_{1}, \mathbf{x}_{2}, \mathbf{x}_{3}\right)$ is attached to

25 the model, where $\mathbf{x}_{3}$ is aligned with the axis of the cylindrical pores. The plane $\left(\mathbf{x}_{1}, \mathbf{x}_{2}\right)$ is the 
1 plane of isotropy for the matrix properties. Given a stiffness tensor $\boldsymbol{c}^{\boldsymbol{m}}$ describing the matrix

2 elasticity, a stiffness tensor $\boldsymbol{c}^{p}$ describing the elasticity of the material in pores, and the volume

3 fraction of pores, a homogenized stiffness tensor $C^{*}$ is calculated using a custom MatLab

$4 \operatorname{code}^{1}$ (The MathWorks, Natick, MA). Since the specimens were kept moist during the

5 measurements, the material in pores (undrained) is assumed to behave like bulk water, that is,

6 bulk modulus and Poisson ratio are set to $2.3 \mathrm{GPa}$ and 0.49 , respectively. Preliminary

7 calculations indicated that computed effective properties are not sensitive to small variations

8 of the elastic properties of the fluid material in pores $\left(c^{p}\right)$. In contrast, they are sensitive to the

9 elastic properties of the matrix $\left(c^{m}\right)$ which must be carefully chosen. In the reference model,

10 we assign the same elastic properties $\boldsymbol{c}^{\boldsymbol{m}}$ and $\boldsymbol{c}^{p}$ to all of the specimens. This amounts to

11 assuming the existence of a "universal" matrix which has yet to be defined. We previously

12 determined [6] the optimal fixed matrix elastic coefficients for the reference model by

13 minimizing the distance between the experimental $\left(C_{\mathrm{ii}}\right)$ and homogenized $\left(C^{*}{ }_{\text {ii }}\right)$ mesoscopic

14 elastic coefficients. Precisely, $\boldsymbol{c}^{\boldsymbol{m}}$ is the tensor which minimizes the objective function defined

15 as:

$16 \quad H_{0}\left(c^{m}\right)=\sqrt{\sum_{k=1}^{N} \sum_{i=1}^{6}\left(\frac{C_{i i ; k}-C_{i i ; k}^{*}\left(c^{m}, c^{p}, P_{k}\right)}{C_{i i i k}}\right)^{2}}$

17 where $N$ is the number of bone specimens, $P_{\mathrm{k}}$ refers to the estimate of porosity of specimen $k$

18 assessed from impedance maps, and $C_{i i ; k}$ and $C_{i i ; k}^{*}$ to its experimental and homogenized

19 elastic coefficients, respectively. Considering all the specimens (i.e. $N=21$ ), the TI stiffness

20 tensor $\boldsymbol{c}^{\boldsymbol{m}}$ which minimizes $H_{0}$ was found to be

$21 \quad c_{11}^{m}=26.8 \mathrm{GPa}, c_{33}^{m}=35.1 \mathrm{GPa}, c_{44}^{m}=7.3 \mathrm{GPa}, c_{66}^{m}=5.8 \mathrm{GPa}$, and $c_{13}^{m}=15.3 \mathrm{GPa}$

22 (These correspond to the following values of engineering moduli $E_{T}^{m}=16.5 \mathrm{GPa}, E_{L}^{m}=24.0$

$23 \mathrm{GPa}, G_{T}^{m}=5.8 \mathrm{GPa}, G_{L}^{m}=7.3 \mathrm{GPa}$ ). This dataset is referred to as the reference matrix

\footnotetext{
${ }^{1}$ Code available on line from www.labos.upmc.fr/lip/spip.php?rubrique133
} 
1 elasticity in the rest of the paper. Values assigned to the bone matrix are consistent with the

2 literature [16-19].

3 In Granke et al. [6], elasticity values predicted with the reference model were calculated to

4 help interpret the data. However, this was without a detailed analysis of the general adequacy

5 of the model, which is the purpose of the present paper.

\section{Discrepancies between data and model predictions}

8 The above values of the mineralized matrix ( $\boldsymbol{c}^{\boldsymbol{m}}$ (Eq. (2)) have been obtained with one specific

9 dataset. In order to ensure that the values are not critically dependent on the dataset, we applied the leave-one-out cross validation (LOOCV) [20, 21]. Ten datasets were formed by excluding the specimens from the $n$-th femur $(n=1 . .10)$ and pooling the specimens from the nine remaining human femurs. For each of these datasets, the elastic tensor $\boldsymbol{c}^{\boldsymbol{m}}\{n\}$ was computed using the objective function defined in Eq (1) (here, $N=18,19$ or 20 depending on the excluded femur $n$ ). The optimized stiffness tensors $c^{m}\{n\}$ for the matrix calculated for the ten datasets were found to be close to the reference matrix as evidenced by the average relative distance between $c^{m}{ }_{i i}\{\mathrm{n}\}$ and $c^{m_{i i}}$ which was less than $1.5 \%$ (Table 1 ), thereby confirming that the reference matrix properties (Eq. 2) are not biased by the particular set of specimens considered in this study.

The adequacy of the fit between the reference model and the experiments was evaluated by means of the root mean square error (RMSE), i.e. the standard deviation of the residuals between the experimental and predicted elastic coefficients. Here, the homogenized stiffness tensor predicted by the reference model for a given specimen harvested from the femur $n$ is computed using $c^{m}\{n\}, c^{p}$, and the porosity of the specimen. Note that the homogenized elasticity $\boldsymbol{C}^{*}$ is thus strictly independent of the mesoscale experimental data $\boldsymbol{C}$. The RMSE absolute and corresponding relative errors were found to be $C_{11}: 1.5 \mathrm{GPa}(7.3 \%), C_{22}: 1.6$ 
$1 \mathrm{GPa}(8.7 \%), C_{33}: 2.0 \mathrm{GPa}(6.6 \%), C_{44}: 0.4 \mathrm{GPa}(6.3 \%) ; C_{55}: 0.5 \mathrm{GPa}(8.5 \%), C_{66}: 0.3 \mathrm{GPa}$

$2(7.9 \%))$.

3

\section{4. Quantification of experimental uncertainties}

5 In this section, we assess whether measurement errors can explain the deviation between

6 experimental observations and model predictions.

\subsection{Porosity and elasticity}

8 Longitudinal $\left(C_{11}, C_{22}, C_{33}\right)$ and shear $\left(C_{44}, C_{55}, C_{66}\right)$ elastic coefficients were obtained by

9 processing longitudinal and shear ultrasound velocity measurements, which lead to different

10 experimental errors for longitudinal and shear coefficients. The measurement relative error $E^{C}$

11 (repeatability) is $3.2 \%$ and $4.7 \%$ for the longitudinal and shear elastic coefficients,

12 respectively [6]. The standard deviations corresponding to these errors were calculated for

13 each coefficient and exhibited the following maximal values: $0.7 \mathrm{GPa}$ for $C_{11}$ and $C_{22}, 1.1$

$14 \mathrm{GPa}$ for $C_{33}, 0.3 \mathrm{GPa}$ for $C_{44}$ and $C_{55}$, and $0.2 \mathrm{GPa}$ for $C_{66}$.

15 As for the error on the porosity estimate $P$, the comparison on ten specimens between $P$ and

16 the volumetric porosity obtained from SR- $\mu \mathrm{CT}$ (taken as a reference) led to an average error

17 of $E^{P}=0.8 \%$ point of porosity [6].

18 When taking into account the measurement errors, we considered that: i) the actual

19 experimental elastic coefficient lies within $\Delta C=\left[\left(1-E^{C}\right) \cdot C_{\mathrm{ii}},\left(1+E^{C}\right) \cdot C_{\mathrm{ii}}\right]$, where $C_{\mathrm{ii}}$ is the

20 experimentally measured elastic coefficient and $E^{C}=0.032$ and 0.047 for the longitudinal and

21 shear elastic coefficients respectively; and ii) the predicted elastic coefficient, for a specimen

22 with estimated porosity $P$, lies within $\Delta C^{*}=\left[C^{*}{ }_{\mathrm{ii}}\left(\boldsymbol{c}^{m}, \boldsymbol{c}^{p}, P+E^{P}\right), C^{*}{ }_{\mathrm{ii}}\left(\boldsymbol{c}^{\boldsymbol{m}}, \boldsymbol{c}^{p}, P-E^{P}\right)\right]$, where $E^{P}$

$23=0.008$. We found that the ranges $\Delta C$ and $\Delta C^{*}$ overlapped for 72 out of the 126 measured

24 coefficients (Fig. 1). For 19 out of the 21 investigated specimens, there was at least one elastic

25 coefficient for which $\Delta C$ and $\Delta C^{*}$ did not overlap. Based on these results, it can be concluded 
1 that the measurement errors cannot account for the observed discrepancies between

2 experimental and predicted elastic coefficients.

3

$4 \quad 4.2$ Misalignment of the specimen during cutting

5 In the model, the pores and the axis of symmetry of the matrix stiffness are aligned with

6 direction 3. Thus it is assumed that the 1-2-plane defined from the specimen faces after

7 cutting is actually perpendicular to the pores and is the plane of isotropy. However, the

8 specimens faces may not be well aligned with the anatomical axes due to inaccuracy of

9 anatomical landmarks used for the cut. We evaluated the degree of possible misalignment

10 based on the pores orientation observed in longitudinal sections cut from the ten specimens

11 imaged with SR- $\mu \mathrm{CT}$ (see online material). The maximum misalignment was estimated to be

$1210^{\circ}$. The consequence of misalignment is that the stiffness coefficients measured are not

13 precisely the coefficients $C_{\mathrm{ii}}$ on the diagonal of the tensor matrix expressed in the natural basis

14 of the specimen material supposed to be TI. To quantify the error on the experimental

15 assessment of the latter, we compared the diagonal stiffness coefficients of the reference

16 model with a $10^{\circ}$ off-axis deviation of axis 3 to the diagonal stiffness coefficients of the

17 reference model tensor in the natural basis (Fig. 1). The maximum values of the relative

18 variations were $\Delta C^{*}{ }_{11}=1.0 \%, \Delta C^{*}{ }_{33}=1.3 \%, \Delta C_{44}^{*}=1.7 \%$, and $\Delta C_{66}^{*}=1.0 \%$. These values

19 are significantly less than the observed discrepancies between the reference model and

20 experimental points.

21

22 5. Revisiting the model hypotheses

$23 \quad 5.1$ Porosity distribution 
1 In the reference model, any variability of pore shape, size and distribution was disregarded.

2 The influence of these factors was investigated for the subset of ten specimens imaged by SR-

$3 \mu \mathrm{CT}$. We proceeded in two steps:

4 Step 1: Each specimen 3D volume was divided into $\mathrm{N}$ adjacent subvolumes $s v_{\mathrm{k}}(k=1 . . \mathrm{N})$ of

5 approximately $1.5 \times 1.5 \times 1.5 \mathrm{~mm}^{3}$ (Fig. 2a,b). The 3D porosity of each subvolume was

6 calculated from the SR- $\mu \mathrm{CT}$ segmented data. The reference matrix elastic and pore tensors $\boldsymbol{c}^{\boldsymbol{m}}$

7 and $c^{p}$ were assigned to the bone matrix and material in pores phases, respectively. The

8 homogenized elastic tensor $C^{*}$ k was then calculated on each subvolume $s v_{\mathrm{k}}$ using the

9 analytical $\mathrm{AH}$ scheme. The procedure yields a representation of the distribution of millimeter

10 scale elasticity within the specimen. The elastic fluctuations are entirely due to fluctuations of

11 porosity within the specimen (Fig. 2c).

12 Step 2: The second step involved solving the homogenization problem for the whole volume:

13 the homogenized elastic properties of each bone specimen were obtained using finite element

14 computations as in Grimal et al. [1] using a classical procedure [22]. Briefly, the material

15 properties at all points $M(x, y, z)$ that belong to subvolume $s v_{\mathrm{k}}$ were set to be the same constant

$16 C(x, y, z)=C^{*}{ }_{k}$ (Fig. 2d). Six sets of kinematic uniform (KUBC) and stress uniform (SUBC)

17 boundary conditions were applied successively. Stress and strain fields were calculated with a

18 commercial finite element code (COMSOL Multiphysics ${ }^{\circledR} 3.5$ ) in the framework of linear

19 elasticity. The computed apparent stiffness (KUBC) or compliance (SUBC) tensors were

20 obtained by dividing components of strain and stress fields. $C^{S U B C}$ and $C^{K U B C}$ provide lower

21 and upper bounds of the apparent tensor. Note that the computational cost to calculate these

22 bounds without step 1, that is a computation conducted on the entire volume with a mesh so

23 fine as to match the resolution of the $\mathrm{SR} \mu \mathrm{CT}$ images, would have been prohibitively high.

24 With our approach, the convergence of the apparent stiffness tensor computed using an 
1 unstructured mesh of tetrahedral elements was obtained for a mesh composed of about 7000

2 quadratic Lagrange elements with a characteristic size of $700 \mu \mathrm{m}$.

3 The homogenization procedure (steps 1 and 2) was validated by computing the bounds $C^{S U B C}$ 4 and $C^{K U B C}$ on an artificial data set (Fig. 3) corresponding to the reference model, i.e. made of

5 a homogeneous matrix and cylindrical pores organized on a hexagonal periodic pattern for a

6 porosity of $12.5 \%$. The bounds computed with FEM were within $10^{-3} \mathrm{GPa}$ of the theoretical

$7 \quad$ value given by the reference model.

8 The $C^{S U B C}$ and $C^{K U B C}$ bounds computed for the ten bone specimens were found to be very

9 close to the predictions of the reference model: the maximum relative error for the different coefficients were $\Delta C_{11}=1.3 \%, \Delta C_{22}=1.2 \%, \Delta C_{33}=0.8 \%, \Delta C_{44}=1.3 \%, \Delta C_{55}=1.1 \%$ and

$11 \Delta C_{66}=2.4 \%$. This was in spite of the large variations of porosity that are present within some

12 of the specimens, which are typically caused by the presence of large resorption cavities. A

13 striking example is the specimen ' 06 ' for which sub-volumes porosities range from 4 to $37 \%$.

14 In general, the largest discrepancies were found for $C_{11}, C_{22}$ and $C_{66}$, and were more

15 pronounced for those specimens that display large variations of porosity (e.g. specimens '03',

16 '06', '08') (Fig. 3). These results suggest that the spatial distribution of pores sizes and shapes

17 as well as their variations between different specimens, independently of variations of pore

18 volume fraction, are not likely the principal cause of the discrepancies between the reference

19 model predictions and experimental results.

\section{$21 \quad 5.2$ Pore length}

22 The reference model assumes that the pores, representing Haversian channels and resorption 23 cavities, are infinitely long. The fact that the pores are actually finite may be a source of 24 discrepancy between the model predictions and the experiments. Models based on Eshelby's 25 solution for ellipsoidal inclusions in a matrix [23] allow for the consideration of the shape of 
1 the pores, that is, their aspect ratio. Among the possible formulations based on Eshelby's

2 solution, the Mori-Tanaka scheme (MT) appears to be the most relevant [24]. Note that the

3 MT method has been used by several authors to represent bone at the millimeter scale $[9,10$,

4 25-27]. When the ellipsoid in the MT scheme is cylindrical (i.e. the pores are infinitely long),

5 the AH (reference) and MT models yield very close results for the entire range of porosity of

6 cortical bone. (However, it is noteworthy that the AH method offers the advantage of being

7 stable, even at high porosity [24]). Accordingly, we considered a MT model of cortical bone

8 mesoscopic elasticity: the elastic properties of the matrix and pores were defined by the same

9 tensors as for the reference model, respectively $\boldsymbol{c}^{\boldsymbol{m}}$ and $\boldsymbol{c}^{p}$, the inclusions were spheroids, and

10 the aspect ratio $\delta$ (major semi-axis over minor semi-axis) was chosen with regard to the

11 general shape of the pores. In human femoral mid-diaphysis, the osteon length is $4 \mathrm{~mm}$ on

12 average [28]. The diameter of the Haversian canals in women is (mean \pm SD [min-max])

$13150 \pm 119$ [57-457] $\mu \mathrm{m}$ [29]. Accordingly, we assumed that $\delta$ resides in the range 10-70.

14 Computations showed that the MT effective elastic properties change only very slightly when

15 increasing the aspect ratio beyond 10 (solid line in Fig. 4) suggesting that aspect ratios of

16 ellipsoidal inclusions as small as 10 to 20 can be considered of infinite extent.

\section{Discussion}

Validating models of bone tissue elasticity should consist in a comparison of measured

stiffness tensors and specimen-specific model predictions. In practice, it is difficult to measure all the terms of the stiffness tensor so that only a few elasticity constants are used for

22 the validation: Dong and Guo [8] have used two shear and two longitudinal coefficients,

23 Deuerling et al. [30] and Bauman et al. [31] have used only longitudinal coefficients. In

24 previous works, the specimen-specific model predictions have used a variety of specimen data

25 such as porosity [8, 31], elasticity and areal fractions of osteonal and interstitial tissues [8] and 
1 average orientation of mineral crystals [31]. Here, we have investigated a popular two-phase

2 composite model of cortical bone which predicts the dependency of the mesoscopic elastic

3 coefficients on porosity. The strength of the present study lies in the number of subjects (21

4 specimens from ten female donors), the number of measured and predicted elastic coefficients

5 (three longitudinal and three shear coefficients, although the transverse isotropic model only

6 predicts four different coefficients) and the assessment of Haversian porosity for each

7 specimen.

8

9 We first examined the experimental uncertainties. Although the precision of the experimental

10 data was acceptable, we recognize that it could be improved. The precision of the vascular

11 porosity estimate would increase if calculated from the $3 \mathrm{D}$ volume data, e.g. from a SR- $\mu \mathrm{CT}$

12 scan. Regarding the measurement of elastic properties in human cortical bone, Bernard et al.

13 [32] recently demonstrated the suitability for resonant ultrasound spectroscopy to bring the

14 precision of Young and shear moduli down to about $0.5 \%$. Even though the experimental

15 uncertainties were suboptimal in the present work, they did not account for the observed

16 differences between the measured and predicted elastic coefficients, confirming the need for a

17 close examination of the model assumptions.

19 In the reference model, the vascular porosity was idealized as infinite cylinders of circular

20 cross-section aligned along the bone long axis. We found that, for realistic aspect ratios

21 (length of the pore/diameter of the pore) of Haversian canal, that is, in the range 10-70,

22 modeling the pores as infinite cylinders yields a very good approximation of pores of finite

23 length. Cortical bone is characterized by a gradient of porosity from the endosteal to the

24 periosteal region [12,33] as well as changes in the pores size [29, 34], and the presence of

25 large resorption cavities [28, 35, 36]. We combined AH and FEM in a two-steps scheme to 
1 account for the spatial heterogeneity of the pores distribution which results in fluctuations of

2 millimeter scale elastic properties within the measured specimens of nominal dimensions

$35 \times 5 \times 7 \mathrm{~mm}^{3}$. The results indicated that the details of the distribution of the porosity play a

4 negligible role in the averaged strain and stress distribution at the specimen scale, hence on

5 the values of apparent elastic properties. Using a voxel-based finite element model, Baumann

6 et al. [31] found that a non-uniform spatial distribution of intracortical porosity results in an

7 orthotropic behavior (weaker stiffness in the radial direction as compared to the

8 circumferential, especially towards the epiphyses), which they mainly attributed to the

9 endosteal resorption. We did not observe this phenomenon, likely because we harvested bone

10 specimens that covered the entire cortex but did not include the trabecularized areas (e.g. Fig

$114 \mathrm{~A}$ in [37]), i.e. the large resorption cavities typically present on the endosteal surface.

12 Interestingly, the model gives a satisfactory prediction of the variations of millimeter-scale

13 elastic coefficients by assuming that the porosity variations between samples are due to

14 changes of either diameter or number of cylindrical pores aligned with the bone axis (the

15 analytical model does not make any distinction between these two options). It should be noted

16 that the analytical model disregards the network of pores perpendicular to the bone axis

17 (Volkmann's canals), while the FE model takes the real shape and distribution of the vascular

18 pores and resorption lacunae into account. The question why it is possible to obtain

19 satisfactory predictions without explicitly modeling the Volkmann's canals was not in the

20 scope of this study. However, one reason could be that cortical tissue in long bones contains

21 much more Haversian canals than Volkmann canals. Therefore, the variability in overall pore

22 volume fraction can be assumed to be dominated by variations in the Haversian canals

23 network. It is of course possible to build a model that accounts separately for pores aligned

24 and perpendicular to the bone axis, which will allow to address this question in future studies. 
1 The predictions of the model critically depend on the assumed values of the mineralized

2 matrix stiffness. Using data from the literature is questionable as bone matrix elastic

3 properties can be significantly different depending on the cortical site [38], the specimen

4 preparation [39-41] or the spatial resolution of the probing technique (e.g. the penetration

5 depth in nanoindentation testing $[42,43]$ or the lateral dimension of the ultrasound beam in

6 SAM [44]). Moreover, most of experimental bone studies do not provide the full stiffness

7 tensor but only elastic properties in one direction (along the osteons axial direction) and they

8 usually discriminate between osteonal and interstitial tissue instead of providing average

9 elastic properties for the bone matrix. To the best of our knowledge, there is no study

10 reporting the anisotropic elastic properties for native matrix tissue from a human femoral mid-

11 diaphysis.

12 In the present work, the model assumes fixed stiffness coefficients $\left(\boldsymbol{c}^{\boldsymbol{m}}\right)$ for the bone matrix.

13 However, the elastic properties of the matrix in human femoral bone are susceptible to

14 change, among other factors, with age [45] and anatomical location [19]. Physiological

15 variations of elasticity at the microscopic level have been documented to range between 5 and

$1615 \%[19,45]$. Note that this was the case for the specimens used in this study [6]: precisely,

17 the conversion of the acoustic impedance values into elastic coefficients [46] led to intra-

18 specimens elastic variations of 8 to $10.5 \%$.

19 We found that the discrepancies between model predictions and experimental data can neither

20 be explained by experimental errors nor by the detailed shape and distribution of the pores.

21 Individual variation of matrix elasticity is one factor which warrants further studies. As a first

22 step, we propose to assess the sensitivity of effective stiffness coefficients to matrix stiffness

23 controlled variations. We computed solutions for two sets of matrix coefficients defined as a \pm

$2410 \%$ variation of the reference values (Eq. (2)). The associated variations of the predicted

25 effective elasticity (averaged over the entire measured porosity range) are $\Delta C^{*}{ }_{11}= \pm 1.8 \mathrm{GPa}$, 
$1 \Delta C_{33}^{*}= \pm 2.8 \mathrm{GPa}, \Delta C^{*}{ }_{44}= \pm 0.6 \mathrm{~Pa}, \Delta C_{66}^{*}= \pm 0.4 \mathrm{GPa}$. Hence, the effective elasticity

2 variations due to small $( \pm 10 \%)$ matrix property variations are likely larger than the

3 experimental uncertainties and consistent with the range of fluctuations of the experimental

4 data (Fig. 5). This result suggests that a precise specimen-specific model of a cortical bone

5 specimen should account for specimen-specific matrix elastic properties, which are likely to

6 vary, for example, with changes in tissue mineral content [47-49] and average orientation of

7 mineral fibrils $[30,50]$.

8 While a simple scaling of all elastic coefficients was sufficient to test for the influence of the

9 matrix elasticity, this approach remains too simplistic for an accurate specimen-specific

10 model (e.g. an increase in the axial stiffness $c_{33}^{m}$ may not necessarily be associated to an

11 increase in $\left.c_{11}^{m}\right)$. Hence, the validation of a proper model of the matrix properties and their

12 variations appears as a natural perspective of this work.

13 Multistep homogenization schemes can be used to derive the stiffness tensor of matrix

14 elasticity, starting from the physical properties of bone constituents (collagen, water, mineral)

$15[9,25,26,51]$. Upon assuming certain composition and organization rules for the different

16 phases, it may be possible to obtain a transversely isotropic stiffness tensor with less than five

17 degrees of freedom $[10,52]$. Modeling of cortical bone material properties at the millimeter-

18 scale with a two-phase model is a framework that was used here for elastic modeling. It is

19 worth noting that strength [53], viscoelasticity [54], and poroelasticity [55] of cortical bone

20 may also be explained in this framework.

22 A limitation of the study is the unique anatomical origin of our specimens which were all

23 harvested in the femoral mid-diaphysis, thereafter exhibiting transversely isotropic elastic

24 properties, in agreement with previous studies $[11,56]$. The application of the proposed

25 model to anatomical sites which can reveal an apparent orthotropic elastic behavior is not 
1 straightforward (e.g. near the femoral or tibial epiphyses [57, 58]). Further investigations are

2 needed to clarify the respective contributions of the matrix elasticity symmetry and the pore

3 network to the orthotropic behavior and consequently adapt the model. Finally, future studies

4 should include a larger number of specimens and/or a higher precision to distinguish between

5 the discrepancies that can be attributed respectively to experimental noise and matrix

6 elasticity.

8 Conclusion

9 In this work we compared model predictions of effective stiffness with experimental data on 10 human cortical bone specimens. Although most of the models that have been proposed for 11 cortical bone were based on several steps of homogenization and a large number of variable 12 parameters, the careful comparison conducted here between experimental data and model

13 predictions support our hypothesis that a relatively simple model, namely a two-phase 14 composite material, is a suitable representation for cortical bone. Several factors may in 15 principle have an effect on millimeter-scale elastic properties: relative fractions of osteonal 16 and interstitial tissues, osteon types associated with different patterns of fibril orientations, 17 volume fractions and shapes of porosities at the different hierarchy levels, quality and volume 18 fractions of mineral and collagen molecules, etc. The results presented in this paper support 19 the validity of the two-phase composite material model of cortical bone which assumes that 20 the essential source of variations of elastic properties at the millimeter-scale is the volume 21 fraction of Haversian porosity. We propose that the bulk of the remaining discrepancies 22 between predicted stiffness coefficients and experimental data (RMSE between 6\% and 9\%) 23 is for a part due to experimental errors and for another part due to small variations of the 24 extravascular matrix properties. 
1 The outcome of this study provides valuable insights for predicting the variations of bone

2 elasticity at the millimeter scale. Ultimately, a simple and accessible model that can reliably

3 predict changes of anisotropic elasticity would be a useful tool for the bone community, e.g.

4 to feed finite element models commonly used in fracture risk assessment or orthopaedics

5 (implant development, preoperative planning) or to investigate structure-functional

6 relationships (effect of bone remodeling on local elasticity).

7 Future in vitro studies may consider including an individualized matrix elasticity in order to

8 obtain a model specific to a given cortical bone specimen. For in vivo applications, there is, to

9 date, no clinical tool allowing for the assessment of matrix elasticity from a patient's bone.

10 However, implementing the proposed model (with fixed matrix properties) in subject-specific

11 FE analyses would be straightforward. This could be done directly from CT data in a similar

12 manner as described in the work of Hellmich et al. [59], i.e. by converting the pore volume

13 fraction of each voxel (deduced from its Hounsfield Unit value) into the corresponding

14 anisotropic elastic tensor using the two-phase micromechanical model presented in this work.

15 Such implementation would constitute a step forward in improving bone mechanical behavior

16 predictions as it overcomes one of the main flaws of current subject-specific FE models, that

17 is, material properties are frequently assumed to be isotropic [60].

18 Additionally, another class of problems that can benefit from the present work are finite

19 difference time-domain (FDTD) simulations aiming at elucidating the interaction mechanisms

20 between ultrasound and bone structures [61].

22 Acknowledgements This work has been conducted within the European Associated

23 Laboratory "Ultrasound Based Assessment of Bone" (ULAB) funded by CNRS (France). It

24 was also supported by the ESRF Long Term Proposal MD431 and the Deutsche

25 Forschungsgemeinschaft within the priority program SPP1420 'Biomimetic Materials 
1 Research: Functionality by Hierarchical Structuring of Materials' (grants Ra1380/7-1 and

2 Ge1894/3-1). W. Parnell is grateful to the Engineering and Physical Sciences Research

3 Council (EPSRC) for funding via grant EP/H01011/1. 


\section{References}

[1] Grimal Q, Raum K, Gerisch A, Laugier P. A determination of the minimum sizes of representative volume elements for the prediction of cortical bone elastic properties.

Biomechanics and modeling in mechanobiology 2011;10:925-37.

[2] Wachter NJ, Krischak GD, Mentzel M, Sarkar MR, Ebinger T, Kinzl L, et al. Correlation of bone mineral density with strength and microstructural parameters of cortical bone in vitro. Bone 2002;31:90-5.

[3] Rho JY, Zioupos P, Currey JD, Pharr GM. Microstructural elasticity and regional heterogeneity in human femoral bone of various ages examined by nano-indentation. Journal of biomechanics 2002;35:189-98.

[4] Dong XN, Guo XE. The dependence of transversely isotropic elasticity of human femoral cortical bone on porosity. Journal of biomechanics 2004;37:1281-7.

[5] McCalden RW, McGeough JA, Barker MB, Court-Brown CM. Age-related changes in the tensile properties of cortical bone. The relative importance of changes in porosity, mineralization, and microstructure. The Journal of bone and joint surgery American volume 1993; 75:1193-205.

[6] Granke M, Grimal Q, Saied A, Nauleau P, Peyrin F, Laugier P. Change in porosity is the major determinant of the variation of cortical bone elasticity at the millimeter scale in aged women. Bone 2011;49:1020-6.

[7] Crolet JM, Aoubiza B, Meunier A. Compact bone: numerical simulation of mechanical characteristics. Journal of biomechanics 1993;26:677-87.

[8] Dong XN, Guo XE. Prediction of cortical bone elastic constants by a two-level micromechanical model using a generalized self-consistent method. J Biomech Eng 2006;128:309-16.

[9] Hellmich C, Ulm FJ, Dormieux L. Can the diverse elastic properties of trabecular and cortical bone be attributed to only a few tissue-independent phase properties and their interactions? Arguments from a multiscale approach. Biomechanics and modeling in mechanobiology 2004;2:219-38.

[10] Grimal Q, Rus G, Parnell WJ, Laugier P. A two-parameter model of the effective elastic tensor for cortical bone. Journal of biomechanics 2011;44:1621-5.

[11] Yoon HS, Katz JL. Ultrasonic wave propagation in human cortical bone--II. Measurements of elastic properties and microhardness. Journal of biomechanics 1976;9:45964.

[12] Saied A, Raum K, Leguerney I, Laugier P. Spatial distribution of anisotropic acoustic impedance assessed by time-resolved 50-MHz scanning acoustic microscopy and its relation to porosity in human cortical bone. Bone 2008;43:187-94.

[13] Raum K. Microelastic imaging of bone. IEEE transactions on ultrasonics, ferroelectrics, and frequency control 2008;55:1417-31.

[14] Parnell WJ, Abrahams ID. Homogenization for wave propagation in periodic fibrereinforced media with complex microstructure. I-Theory. J Mech Phys Solids 2008;56:2521-40.

[15] Parnell WJ, Grimal Q. The influence of mesoscale porosity on cortical bone anisotropy. Investigations via asymptotic homogenization. Journal of the Royal Society, Interface / the Royal Society 2009;6:97-109.

[16] Turner CH, Rho J, Takano Y, Tsui TY, Pharr GM. The elastic properties of trabecular and cortical bone tissues are similar: results from two microscopic measurement techniques. Journal of biomechanics 1999;32:437-41.

[17] Rho JY, Roy ME, Tsui TY, Pharr GM. Elastic properties of microstructural components of human bone tissue as measured by nanoindentation. J Biomed Mater Res 1999;45:48-54. 
[18] Fan Z, Swadener JG, Rho JY, Roy ME, Pharr GM. Anisotropic properties of human tibiae cortical bone as measured by nanoindentation. J Orthop Res 2002;20:806-10.

[19] Rohrbach D, Lakshmanan S, Peyrin F, Langer M, Gerisch A, Grimal Q, et al. Spatial distribution of tissue level properties in a human femoral cortical bone. Journal of biomechanics 2012;45:2264-70.

[20] Stone M. Cross-Validatory Choice and Assessment of Statistical Predictions. J R Stat Soc B 1974;36:111-47.

[21] Geisser S. The Predictive Sample Reuse Method with Applications. Journal of the American Statistical Association 1975;70:320-8.

[22] Zohdi T, Wriggers P. An Introduction to Computational Micromechanics. Berlin: Springer-Verlag; 2005.

[23] Eshelby JD. The Determination of the Elastic Field of an Ellipsoidal Inclusion, and Related Problems. Proc R Soc A 1957;241:376-96.

[24] Parnell WJ, Vu MB, Grimal Q, Naili S. Analytical methods to determine the effective mesoscopic and macroscopic elastic properties of cortical bone. Biomechanics and modeling in mechanobiology 2012;11:883-901.

[25] Hamed E, Lee Y, Jasiuk I. Multiscale modeling of elastic properties of cortical bone. Acta Mechanica 2010;213:131-54.

[26] Martinez-Reina J, Dominguez J, Garcia-Aznar JM. Effect of porosity and mineral content on the elastic constants of cortical bone: a multiscale approach. Biomechanics and modeling in mechanobiology 2011;10:309-22.

[27] Sansalone V, Bousson V, Naili S, Bergot C, Peyrin F, Laredo JD, et al. Anatomical distribution of the degree of mineralization of bone tissue in human femoral neck: impact on biomechanical properties. Bone 2012;50:876-84.

[28] Cooper DM, Thomas CD, Clement JG, Hallgrimsson B. Three-dimensional microcomputed tomography imaging of basic multicellular unit-related resorption spaces in human cortical bone. The anatomical record Part A, Discoveries in molecular, cellular, and evolutionary biology 2006;288:806-16.

[29] Cooper DM, Thomas CD, Clement JG, Turinsky AL, Sensen CW, Hallgrimsson B. Agedependent change in the 3D structure of cortical porosity at the human femoral midshaft. Bone 2007;40:957-65.

[30] Deuerling JM, Yue W, Espinoza Orias AA, Roeder RK. Specimen-specific multi-scale model for the anisotropic elastic constants of human cortical bone. Journal of biomechanics 2009;42:2061-7.

[31] Baumann AP, Deuerling JM, Rudy DJ, Niebur GL, Roeder RK. The relative influence of apatite crystal orientations and intracortical porosity on the elastic anisotropy of human cortical bone. Journal of biomechanics 2012;45:2743-9.

[32] Bernard S, Grimal Q, Laugier P. Accurate measurement of cortical bone elasticity tensor with resonant ultrasound spectroscopy. Journal of the mechanical behavior of biomedical materials 2013;18:12-9.

[33] Bousson V, Bergot C, Meunier A, Barbot F, Parlier-Cuau C, Laval-Jeantet AM, et al. CT of the middiaphyseal femur: cortical bone mineral density and relation to porosity. Radiology 2000;217:179-87.

[34] Baron C, Talmant M, Laugier P. Effect of porosity on effective diagonal stiffness coefficients (cii) and elastic anisotropy of cortical bone at $1 \mathrm{MHz}$ : a finite-difference time domain study. The Journal of the Acoustical Society of America 2007;122:1810.

[35] Atkinson PJ. Changes in resorption spaces in femoral cortical bone with age. The Journal of pathology and bacteriology 1965;89:173-8. 
[36] Jordan GR, Loveridge N, Bell KL, Power J, Rushton N, Reeve J. Spatial clustering of remodeling osteons in the femoral neck cortex: a cause of weakness in hip fracture? Bone 2000;26:305-13.

[37] Zebaze RM, Ghasem-Zadeh A, Bohte A, Iuliano-Burns S, Mirams M, Price RI, et al. Intracortical remodelling and porosity in the distal radius and post-mortem femurs of women: a cross-sectional study. Lancet 2010;375:1729-36.

[38] Hoffler CE, Moore KE, Kozloff K, Zysset PK, Brown MB, Goldstein SA. Heterogeneity of bone lamellar-level elastic moduli. Bone 2000;26:603-9.

[39] Zimmerman MC, Prabhakar A, Chokshi BV, Budhwani N, Berndt H. The acoustic properties of normal and imbedded bovine bone as measured by acoustic microscopy. $\mathrm{J}$ Biomed Mater Res 1994;28:931-8.

[40] Bushby AJ, Ferguson VL, Boyde A. Nanoindentation of bone: comparison of specimens tested in liquid and embedded in polymethylmethacrylate. J Mater Res 2004;19:249-59.

[41] Hoffler CE, Guo XE, Zysset PK, Goldstein SA. An application of nanoindentation technique to measure bone tissue Lamellae properties. J Biomech Eng 2005;127:1046-53. [42] Isaksson H, Nagao S, Malkiewicz M, Julkunen P, Nowak R, Jurvelin JS. Precision of nanoindentation protocols for measurement of viscoelasticity in cortical and trabecular bone. Journal of biomechanics 2010;43:2410-7.

[43] Lucchini R, Carnelli D, Ponzoni M, Bertarelli E, Gastaldi D, Vena P. Role of damage mechanics in nanoindentation of lamellar bone at multiple sizes: experiments and numerical modeling. Journal of the mechanical behavior of biomedical materials 2011;4:1852-63.

[44] Raum K, Reisshauer J, Brandt J. Frequency and resolution dependence of the anisotropic impedance estimation in cortical bone using time-resolved scanning acoustic microscopy. Journal of biomedical materials research Part A 2004;71:430-8.

[45] Malo MK, Rohrbach D, Isaksson H, Toyras J, Jurvelin JS, Tamminen IS, et al. Longitudinal elastic properties and porosity of cortical bone tissue vary with age in human proximal femur. Bone 2013;53:451-8.

[46] Preininger B, Checa S, Molnar FL, Fratzl P, Duda GN, Raum K. Spatial-temporal mapping of bone structural and elastic properties in a sheep model following osteotomy. Ultrasound Med Biol 2011;37:474-83.

[47] Raum K, Cleveland RO, Peyrin F, Laugier P. Derivation of elastic stiffness from sitematched mineral density and acoustic impedance maps. Physics in medicine and biology 2006;51:747-58.

[48] Raum K, Leguerney I, Chandelier F, Talmant M, Saied A, Peyrin F, et al. Site-matched assessment of structural and tissue properties of cortical bone using scanning acoustic microscopy and synchrotron radiation muCT. Physics in medicine and biology 2006;51:73346.

[49] Rupin F, Saied A, Dalmas D, Peyrin F, Haupert S, Raum K, et al. Assessment of Microelastic Properties of Bone Using Scanning Acoustic Microscopy: A Face-to-Face Comparison with Nanoindentation. Japanese Journal of Applied Physics 2009;48:07GK1. [50] Zebaze RM, Jones AC, Pandy MG, Knackstedt MA, Seeman E. Differences in the degree of bone tissue mineralization account for little of the differences in tissue elastic properties. Bone 2011;48:1246-51.

[51] Tiburtius S, Schrof S, Molnar F, Varga P, Peyrin F, Grimal Q, et al. On the elastic properties of mineralized turkey leg tendon tissue: multiscale model and experiment.

Biomechanics and modeling in mechanobiology 2014.

[52] Vuong J, Hellmich C. Bone fibrillogenesis and mineralization: quantitative analysis and implications for tissue elasticity. Journal of theoretical biology 2011;287:115-30. 
[53] Fritsch A, Hellmich C, Dormieux L. Ductile sliding between mineral crystals followed by rupture of collagen crosslinks: experimentally supported micromechanical explanation of bone strength. Journal of theoretical biology 2009;260:230-52.

[54] Eberhardsteiner L, Hellmich C, Scheiner S. Layered water in crystal interfaces as source for bone viscoelasticity: arguments from a multiscale approach. Comput Methods Biomech Biomed Engin 2014;17:48-63.

[55] Morin C, Hellmich C. A multiscale poromicromechanical approach to wave propagation and attenuation in bone. Ultrasonics 2014;54:1251-69.

[56] Espinoza Orias AA, Deuerling JM, Landrigan MD, Renaud JE, Roeder RK. Anatomic variation in the elastic anisotropy of cortical bone tissue in the human femur. Journal of the mechanical behavior of biomedical materials 2009;2:255-63.

[57] Rho JY. An ultrasonic method for measuring the elastic properties of human tibial cortical and cancellous bone. Ultrasonics 1996;34:777-83.

[58] Rudy DJ, Deuerling JM, Espinoza Orias AA, Roeder RK. Anatomic variation in the elastic inhomogeneity and anisotropy of human femoral cortical bone tissue is consistent across multiple donors. Journal of biomechanics 2011;44:1817-20.

[59] Hellmich C, Kober C, Erdmann B. Micromechanics-based conversion of CT data into anisotropic elasticity tensors, applied to FE simulations of a mandible. Annals of biomedical engineering 2008;36:108-22.

[60] Yosibash Z, Tal D, Trabelsi N. Predicting the yield of the proximal femur using highorder finite-element analysis with inhomogeneous orthotropic material properties. Philosophical transactions Series A, Mathematical, physical, and engineering sciences 2010;368:2707-23.

[61] Bossy E, Grimal Q. Numerical Methods for Ultrasonic Bone Characterization. In: Laugier P, Haïat G, editors. Bone Quantitative Ultrasound: Springer Netherlands; 2011. p. 181-228.

\section{Tables}

Table 1 Optimized stiffness tensor for the matrix properties calculated after considering different sets of specimens

\section{Figures captions}

Fig. 1 Longitudinal (left) and shear (right) elastic coefficients versus porosity. The solid lines display the elastic coefficients computed with the reference model. The dotted lines show the influence of a $10^{\circ}$ off-axis deviation of axis 3 . Boxes represent the experimental errors on the measurement of elastic coefficients $C_{\mathrm{ii}}$ and the evaluation of porosity. The boxes highlighted in red indicate those measurement errors which cannot entirely explain the distance between the experimental and predicted elastic coefficients (54 out of 126 measured coefficients).

Fig. 2 (a,b) The bone volume is divided into subvolumes of approximately $1.5 \mathrm{~mm}$. (c) The $3 \mathrm{D}$ porosity of each subvolume is calculated from the SR- $\mu \mathrm{CT}$ segmented data. The homogenized elastic tensor of each subvolume is computed using asymptotic homogenization. (d) Finite element modeling on the bone specimen. The coordinatedependent material properties are retrieved from the homogenized elastic tensors calculated 
on each subvolume. Applying a set of uniform boundary conditions allows one to assess the lower and upper bounds of the apparent elastic coefficients.

Fig. 3 Homogenized elastic properties: reference model (pore volume fraction) versus Finite Element Model (real pore shape and distribution taken into account). Illustration of the coefficient $\mathrm{C}_{11}$ for an artificial dataset that corresponds to the reference model (i.e. made of a homogeneous matrix and cylindrical pores organized on a hexagonal periodic pattern) and 10 human bone specimens.

Fig. 4 Homogenized elastic coefficients as obtained from the Mori-Tanaka (MT) model (solid lines) versus the pore aspect ratio (= length/diameter) for a given porosity of $15 \%$. The effective elastic coefficients as obtained with the reference model (dotted lines) have been superimposed. The grey zone represents the range of aspect ratios of Haversian canals in femoral human cortical bone.

Fig. 5 Relative differences between experimental data and the predictions of the reference model versus porosity. The solid horizontal lines correspond to the reference model. The dotted lines correspond to the predictions of the model with modified matrix elastic coefficients ( $\pm 10 \%$ starting from the reference model values) 

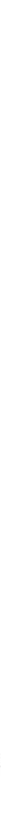

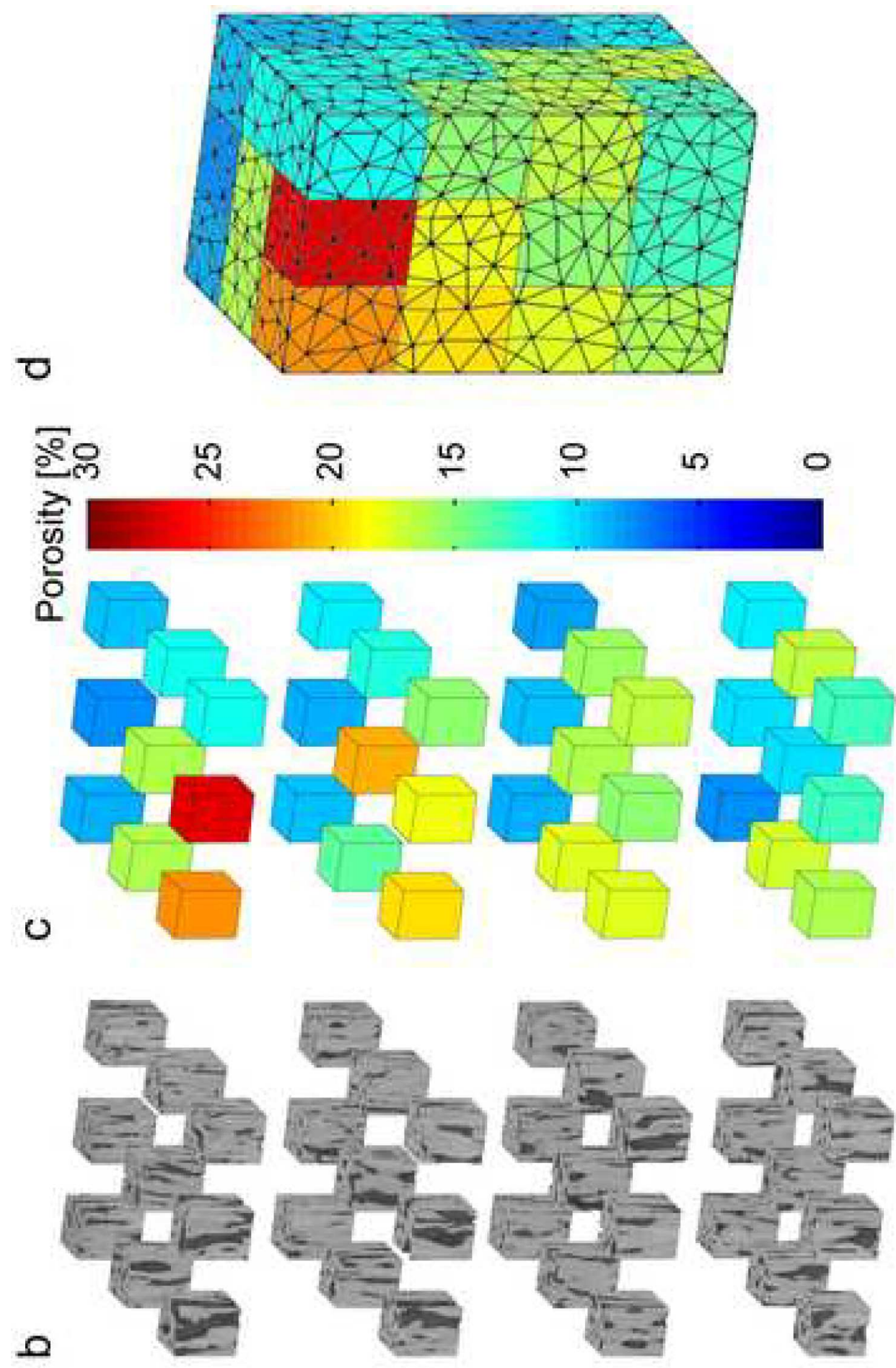

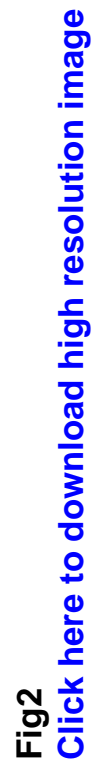

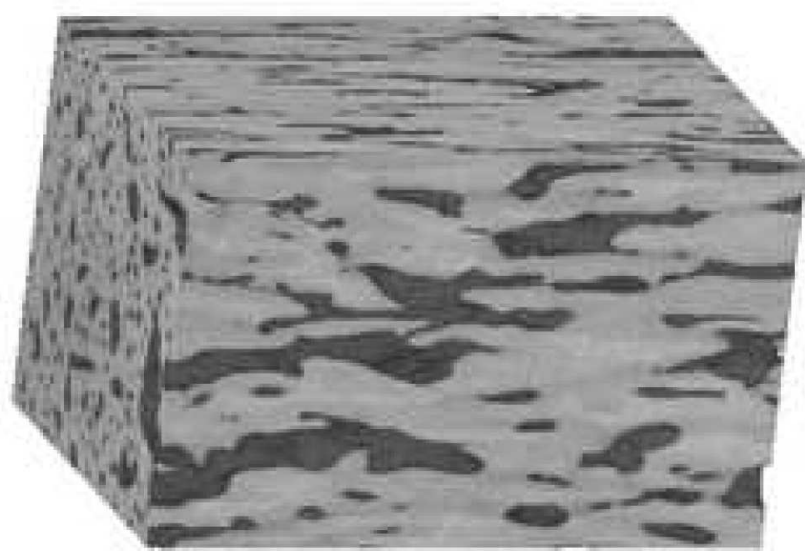


Pore distribution not taken into account

$25 \square$ Reference model

24

Pore distribution taken into account

23 FE model lower bound

22

FE model upper bound

$\frac{\pi}{0}$

21

20

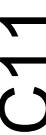

19

18

17

16

15

Artificia dataset

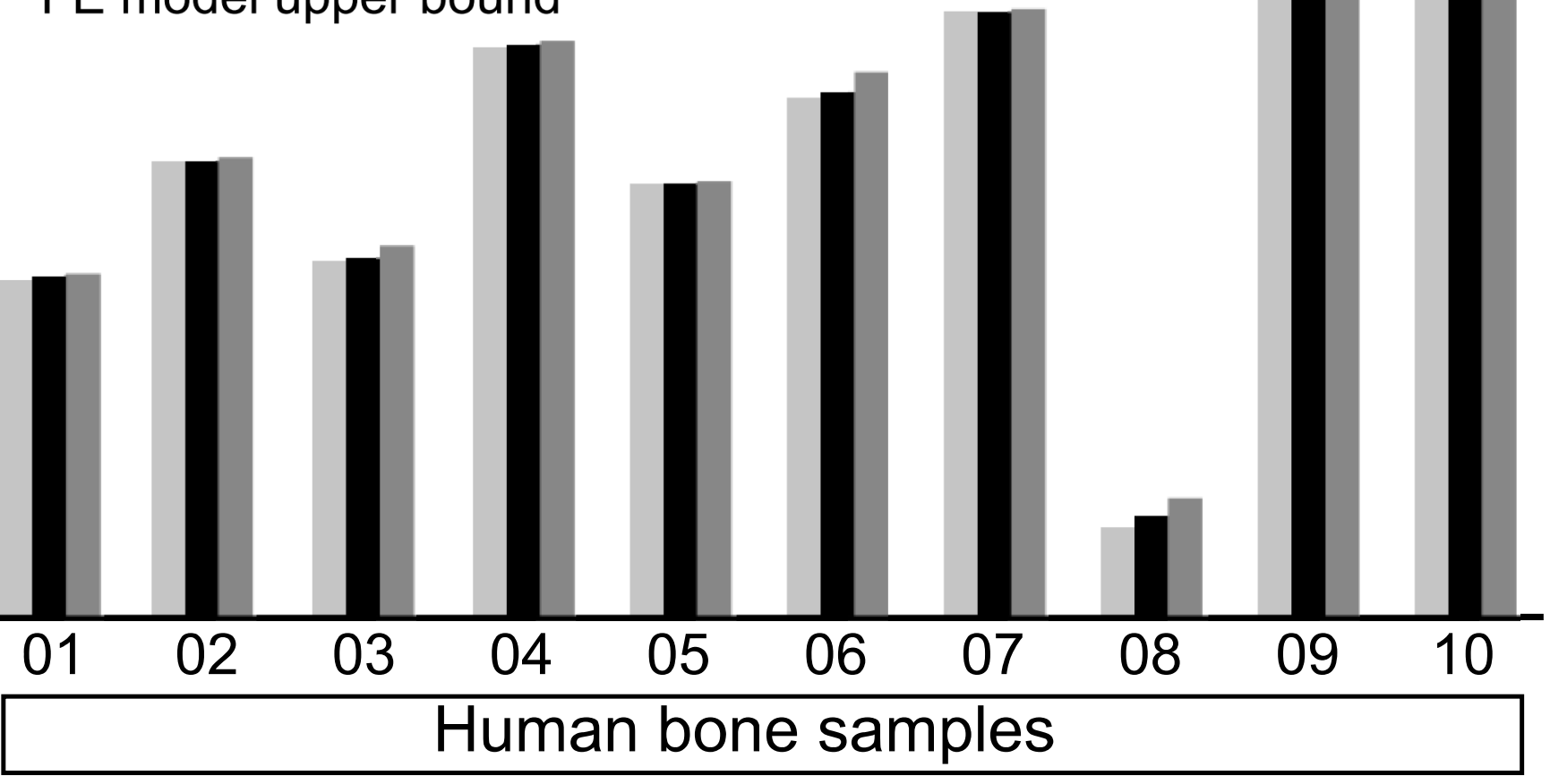




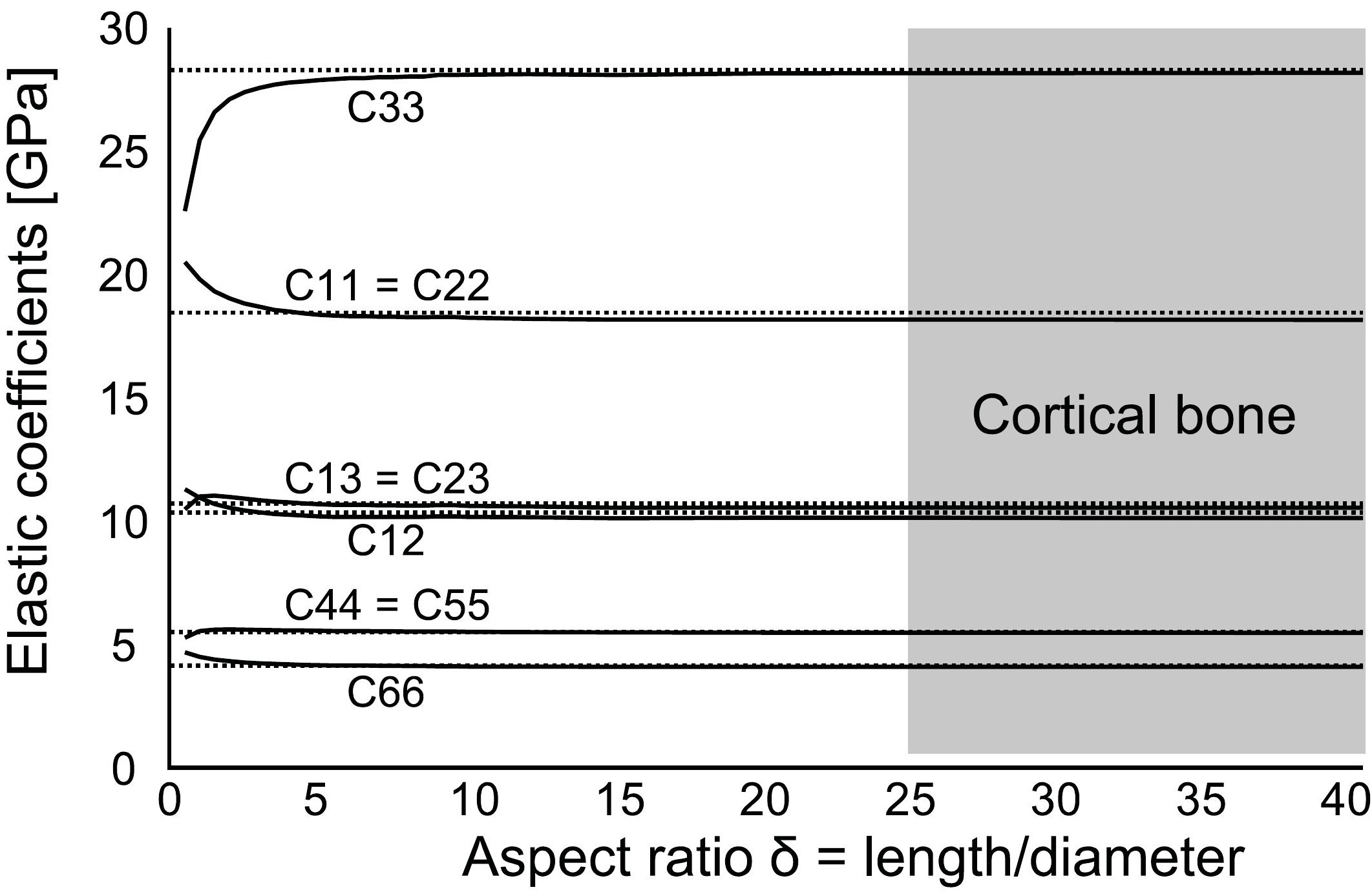



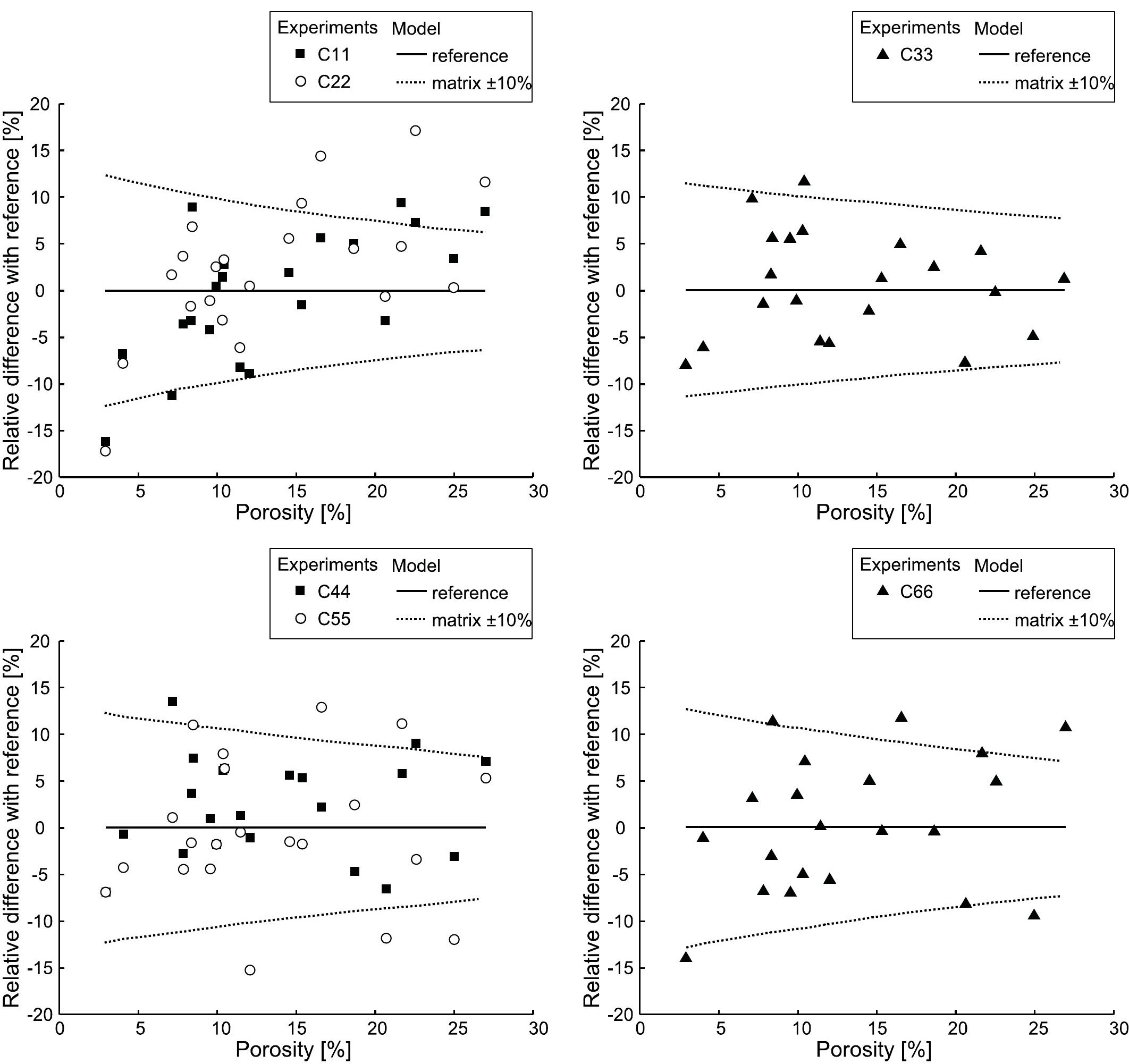


\begin{tabular}{c|ccccc} 
Excluded femur (matrix elastic tensor) & $c_{11}^{m}[\mathrm{GPa}]$ & $c_{33}^{m}[\mathrm{GPa}]$ & $c_{44}^{m}[\mathrm{GPa}]$ & $c_{66}^{m}[\mathrm{GPa}]$ & $c_{13}^{m}[\mathrm{GPa}]$ \\
\hline None $\left(\boldsymbol{c}^{\boldsymbol{m}}, \boldsymbol{r e f e r e n c e ~ m o d e l}\right)$ & 26.8 & 35.1 & 7.3 & 5.8 & 15.4 \\
\hline$\# 218\left(\boldsymbol{c}^{\boldsymbol{m}}\{1\}\right)$ & 26.6 & 35.2 & 7.2 & 5.7 & 15.7 \\
\hline$\# 227\left(\boldsymbol{c}^{\boldsymbol{m}}\{2\}\right)$ & 26.8 & 35.2 & 7.3 & 5.8 & 15.6 \\
\hline$\# 228\left(\boldsymbol{c}^{\boldsymbol{m}}\{3\}\right)$ & 26.9 & 35.3 & 7.3 & 5.8 & 15.6 \\
\hline$\# 245\left(\boldsymbol{c}^{\boldsymbol{m}}\{4\}\right)$ & 26.6 & 34.9 & 7.2 & 5.7 & 15.4 \\
\hline$\# 251\left(\boldsymbol{c}^{\boldsymbol{m}}\{5\}\right)$ & 26.9 & 34.4 & 7.2 & 5.8 & 13.7 \\
\hline$\# 260\left(\boldsymbol{c}^{\boldsymbol{m}}\{6\}\right)$ & 26.8 & 34.6 & 7.3 & 5.8 & 14.3 \\
\hline$\# 263\left(\boldsymbol{c}^{\boldsymbol{m}}\{7\}\right)$ & 26.6 & 35.2 & 7.3 & 5.7 & 15.7 \\
\hline$\# 267\left(\boldsymbol{c}^{\boldsymbol{m}}\{8\}\right)$ & 27.3 & 37.5 & 7.3 & 5.8 & 21.0 \\
\hline$\# 268\left(\boldsymbol{c}^{\boldsymbol{m}}\{9\}\right)$ & 26.9 & 34.9 & 7.4 & 5.8 & 13.5 \\
\hline$\# 271\left(\boldsymbol{c}^{\boldsymbol{m}}\{10\}\right)$ & 26.6 & 34.8 & 7.3 & 5.8 & 13.6 \\
\hline mean \pm std & $\mathbf{2 6 . 8} \pm \mathbf{0 . 2}$ & $\mathbf{3 5 . 2} \pm \mathbf{0 . 9}$ & $\mathbf{7 . 3} \pm \mathbf{0 . 0 4}$ & $\mathbf{5 . 8} \pm \mathbf{0 . 0 3}$ & $\mathbf{1 5 . 4} \pm \mathbf{2 . 2}$ \\
\hline
\end{tabular}




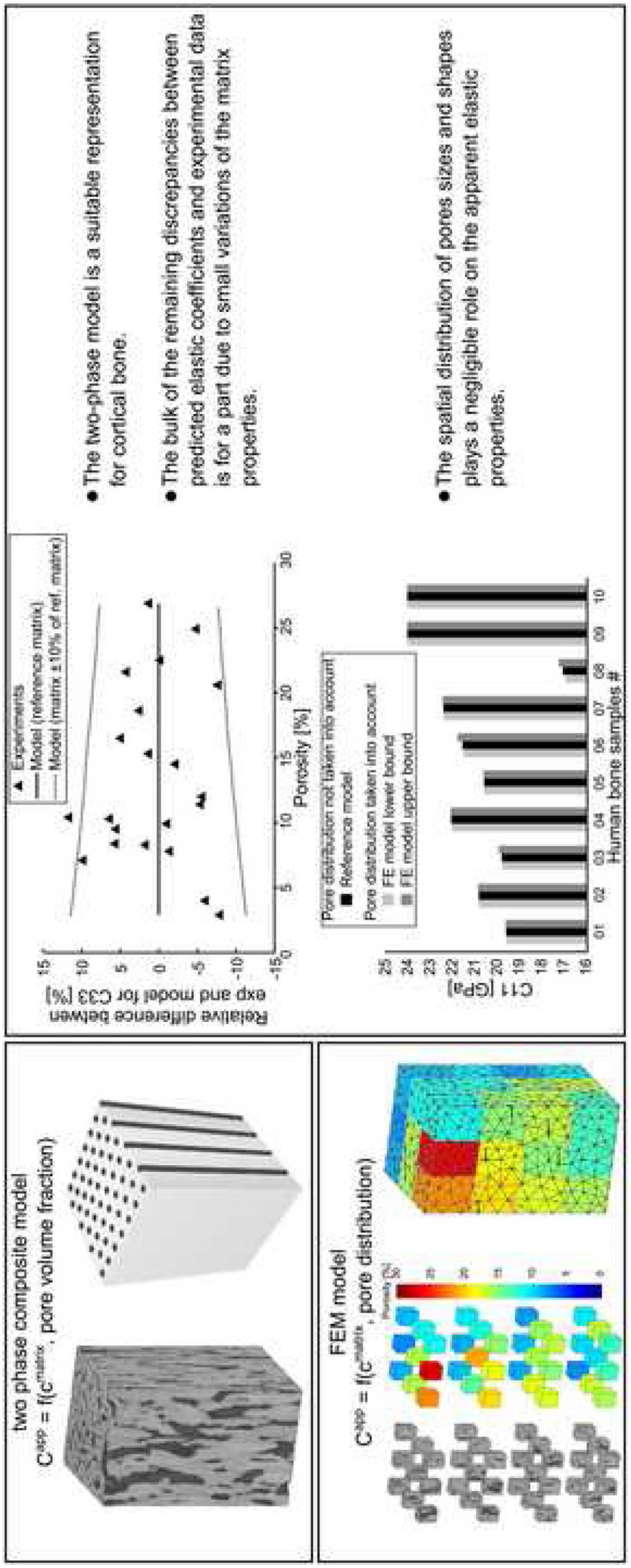

